\title{
Size-dependent catalytic effect of magnetite nanoparticles in the synthesis of tunable magnetic polyaniline nanocomposites
}

\author{
$4 \quad$ Ermelinda Falletta $^{1^{*}}$, Anna M. Ferretti ${ }^{2}$, Sara Mondini ${ }^{2}$, Claudio Evangelisti ${ }^{2 \dagger}$, Elena \\ Capetti $^{2}$, Elena Sonia Olivetti ${ }^{3}$, Luca Martino ${ }^{3}$, Cinzia Beatrice ${ }^{3}$, Gabriel Soares ${ }^{3}$, \\ Massimo Pasquale ${ }^{3}$, Cristina Della Pina ${ }^{1}$, Alessandro Ponti ${ }^{2 *}$ \\ ${ }^{1}$ Department of Chemistry, University of Milan, via C. Golgi, 19, 20133, Milan (Italy) \\ $8 \quad 2$ Istituto di Scienze e Tecnologie Chimiche "Giulio Natta” (SCITEC), Consiglio Nazionale \\ delle Ricerche, via G. Fantoli 16/15, 20138, Milan (Italy) \\ ${ }^{3}$ Istituto Nazionale di Ricerca Metrologica (INRIM), Strada delle Cacce 91, 10135, Torino
} (Italy)

13 *Corresponding author, e-mail: alessandro.ponti@scitec.cnr.it, ermelinda.falletta@unimi.it 14

$15 \dagger$ Present address: Istituto di Chimica dei Composti Organometallici (ICCOM), Consiglio 16 Nazionale delle Ricerche, Via G. Moruzzi 1, 56124, Pisa (Italy)

17

19 Nanocomposites comprising magnetic nanoparticles (NPs) embedded in an organic conducting 20 polymer are promising materials that may allow one to exploit synergic effects between the 21 electrically conducting and the magnetically permeable components. Having already shown 22 that magnetite NPs can be conveniently used as a catalyst for the oxidative polymerization of 23 the aniline dimer resulting in NPs embedded in the final composite and how to modulate the 24 magnetic coercivity of the composites, we now turn to investigate how the size of magnetite 25 NPs affects the polymerization and the properties of the final composite. Magnetite NPs of 26 diameter $2.3,10$, and $27 \mathrm{~nm}$ turned out to be effective catalysts with cheap oxidants such as $27 \mathrm{H}_{2} \mathrm{O}_{2}$ and $\mathrm{O}_{2}$. Yield data show that the rate-determining step occurs on the NP surface. 28 Extensive characterization shows that the NPs are well-dispersed in the composite with no 29 significant morphological change. The static magnetic properties of the composites are widely 30 different, e. g, the magnetic blocking temperature shifts from $290 \mathrm{~K}$ for $27 \mathrm{~nm}$ NPs to $54 \mathrm{~K}$ for $3110 \mathrm{~nm}$ NPs while composites with $2.3 \mathrm{~nm}$ NPs are virtually unblocked down to $5 \mathrm{~K}$. The 32 dynamic electromagnetic behavior studied up to the microwave range only shows energy 33 absorptions associated to the ferromagnetic resonance, at frequencies around $1 \mathrm{GHz}$. 
35 Keywords: $\mathrm{Fe}_{3} \mathrm{O}_{4}$, PANI, magnetic nanoparticles, catalysis, electromagnetic absorption.

\section{Introduction}

39 Since their discovery, intrinsically conducting polymers (CPs) have emerged as outstanding materials for numerous applications in many sectors, ranging from sensors (Wang et al., 2020) to biomedicine (Kaur et al., 2015) passing from organic solar cells (Dong et al., 2012), 2 environmental remediation (Ibanez et al., 2018) and so on (Yang et al., 2019; Li et al., 2020). 43 Among CPs, polyaniline (PANI) is unique for its ease of synthesis, low cost, biocompatibility, environmental stability, and extraordinary tunable properties (Badra et al., 2009).

In recent years, materials combining characteristics and properties of two or more components have been sought and required in many sectors. Among them, $\mathrm{PANI} / \mathrm{Fe}_{3} \mathrm{O}_{4}$ nanocomposites have been recently investigated as advanced materials being both electrically conducting and magnetically permeable and having potential application in fields such as batteries (Wang et al., 2017), electromagnetic interference shielding (Movassagh-Alanagh et al., 2017), 50 environmental remediation (Muhammad et al., 2019), cancer therapy (Ahmadkhani et al., 2019),

52 Several approaches have been developed to prepare these composites: mixing of PANI with $53 \mathrm{Fe}_{3} \mathrm{O}_{4}$, self-assembly method, in situ polymerization, ultrasonic irradiation (Qiu et al., 2006). 54 The ability of magnetite (and cobalt ferrite) nanoparticles (NPs) to act as catalysts in the PANI synthesis was explored for the first time by us in the catalytic polymerization of $\mathrm{N}-4$ 56 (aminophenyl)aniline (Della Pina et al., 2012; Della Pina et al., 2014; Della Pina et al., 2015; 57 Falletta et al., 2015). Most recently, Mišurović and coworkers applied $\mathrm{Fe}_{3} \mathrm{O}_{4} \mathrm{NPs}_{\mathrm{s}}$ as the catalyst in the aniline polymerization (Mišurović et al., 2019).

59 Exploiting magnetite NPs of different size to catalyze the polymerization of AD and prepare $60 \mathrm{PANI} / \mathrm{Fe}_{3} \mathrm{O}_{4}$ nanocomposites would allow one to tune the magnetic properties of the 61 composites provided that the NPs are size monodisperse and effective as a catalyst. The first 62 requirement can be met thanks to the well-established methods of size-controlled colloidal 63 nanochemistry (Ferretti et al., 2021). As to the second one, the activity of a heterogeneous 64 catalysts is strongly related to its particle size since it is intrinsically dependent on the surface65 to-volume ratio, which dramatically grows passing from bulk to nano-dimensions. Moreover, 66 compared to bulk materials, NPs have a larger surface density of atoms in sites, such as corner 67 or edge sites, that maximize their reactivity because NPs are synthesized under kinetic control 
68 and have spherical shape without the well-defined facets or crystals grown near thermodynamic

69 equilibrium. However, because of the small dimensions, the outstanding activity of 70 nanostructured materials is sometimes compromised by their strong interaction with 71 intermediates and by aggregation phenomena. To the best of our knowledge, the size-dependent catalytic activity of $\mathrm{Fe}_{3} \mathrm{O}_{4}$ NPs in the oxidative polymerization of $\mathrm{N}$-4-(aminophenyl)aniline 3 has not yet been investigated. Here, we report our recent results in the production and ${ }_{4}$ characterization of conducting and magnetic $\mathrm{PANI} / \mathrm{Fe}_{3} \mathrm{O}_{4}$ composites exploring at the same time the effect of the $\mathrm{Fe}_{3} \mathrm{O}_{4} \mathrm{NP}$ size on their catalytic activity and the variation of the electromagnetic properties of the materials as function of the NP size.

\section{Experimental}

\section{Materials}

81 Chemicals

2 All chemicals were bought from Merck and used without any purification process. Acetone 83 (Aldrich) was distilled over $\mathrm{KMnO}_{4}$ and stored under argon before use in the synthesis of 2.3 $84 \mathrm{~nm}$ NPs.

85

86 Synthesis of $2.3 \mathrm{~nm}$ magnetite NPs coated with oleic acid and derived from iron solvated metal ions (SMA).

88 The synthesis of Fe-SMA was carried out in a static metal vapor synthesis reactor described 89 elsewhere (Evangelisti et al., 2015) and equipped with an alumina-coated tungsten crucible 90 heated by Joule effect with a generator with a maximum power of $2 \mathrm{~kW}$. Fe-solvated metal 91 atoms (SMA) were prepared according to a previously described procedure (Barbaro et al., 92 2015; Campisi et al., 2019). Briefly, Fe vapors generated in high vacuum $\left(1 \cdot 10^{-5} \mathrm{mbar}\right)$ by 93 resistive heating of an alumina-coated tungsten crucible, filled with approximately $300 \mathrm{mg}$ of 94 iron, were co-condensed at $-196{ }^{\circ} \mathrm{C}$ with acetone vapor $(100 \mathrm{~mL})$ in the glass reactor chamber 95 for $1 \mathrm{~h}$. The reactor chamber was then warmed to the melting point of the solid matrix (ca. -95 $96{ }^{\circ} \mathrm{C}$ ), and the resulting brown Fe-solvated metal atoms (SMA) solution (95 mL) was siphoned 97 at a low temperature into a Schlenk tube under argon atmosphere. Oleic acid $(1 \mathrm{~mL})$ was added 98 to the Fe-SMA at low temperature $\left(-40^{\circ} \mathrm{C}\right)$ under gentle stirring, and then was left overnight at 99 room temperature to the open air. In these conditions, a red-brown precipitate was formed, 100 which was collected by centrifugation. The precipitate was dissolved in $n$-hexane $(50 \mathrm{~mL})$, 101 precipitated with acetone $(50 \mathrm{~mL})$ and collected by centrifugation. This latter procedure was 


\section{Synthesis of $10.9 \mathrm{~nm}$ magnetite NPs coated with oleic acid.}

107 The NPs were synthesized by a modification of a published procedure (Hyeon et al., 2001). 108 Oleic acid $(2.68 \mathrm{~mL}, 8.51 \mathrm{mmol})$ was dissolved in dioctyl ether $(12 \mathrm{~mL})$ in a $100 \mathrm{~mL}$ three-

repeated three times to remove the excess of oleic acid. The solid was then dispersed in toluene $(75 \mathrm{~mL})$. The iron content of the toluene dispersion, measured by the ICP-OES procedure described below, resulted $2.60 \mathrm{~g} / \mathrm{L}$.

neck round-bottom flask, equipped with condenser and thermometer, under magnetic stirring and $\mathrm{N}_{2}$ atmosphere. The solution was subjected to three vacuum- $\mathrm{N}_{2}$ cycles at $\mathrm{RT}$ and then heated to $105^{\circ} \mathrm{C}$. Three vacuum- $\mathrm{N}_{2}$ cycles were carried out at $105^{\circ} \mathrm{C}$. Next, iron pentacarbonyl $(280 \mu 1,2.13 \mathrm{mmol})$ was injected into the solution and the reaction mixture was heated to reflux (ca. $285^{\circ} \mathrm{C}$ ) at a rate of $3{ }^{\circ} \mathrm{C} / \mathrm{min}$ using a programmable heating unit. The solution turned black after $50 \mathrm{~min}$ at reflux and was aged for an additional hour before cooling to RT. The NPs were precipitated from the reaction mixture by adding acetone $(60 \mathrm{~mL})$, separated by centrifugation, and dispersed in petroleum ether $(45 \mathrm{~mL})$. To further purify the NPs, acetone $(60 \mathrm{~mL})$ was added to the NP dispersion and the NPs were collected by centrifugation and dispersed in toluene $(23 \mathrm{~mL})$. The iron content of the toluene dispersion, measured by the UV-Vis procedure described below, was $9.7 \mathrm{~g} / \mathrm{L}$.

\section{Synthesis of $26.0 \mathrm{~nm}$ magnetite NPs coated with oleic acid.}

The NPs were synthesized by a modification of a published procedure (Park et al., 2004). The metal precursor $\mathrm{Fe}(\mathrm{III})$ trioleate $\left(\mathrm{FeOl}_{3}\right)$ was synthesized as follows. Potassium oleate $(5.77 \mathrm{~g}$., $18 \mathrm{mmol})$ and $\mathrm{FeCl}_{3} \cdot 6 \mathrm{H}_{2} \mathrm{O}$ were dissolved in a mixture of $\mathrm{H}_{2} \mathrm{O}(10 \mathrm{~mL})$ and $\mathrm{EtOH}(12 \mathrm{~mL})$ in a $100 \mathrm{~mL}$ round-bottom flask under magnetic stirring and $\mathrm{N}_{2}$ atmosphere. Hexane $(21 \mathrm{~mL})$ was added to the reaction mixture, which was then heated to $70{ }^{\circ} \mathrm{C}$ for $4 \mathrm{~h}$. After cooling to RT, the mixture was transferred to a $100 \mathrm{~mL}$ separatory funnel and the aqueous phase discarded. The red-brown organic phase was washed with deionized water $(2 \times 10 \mathrm{~mL})$ and with saturated aqueous $\mathrm{NaCl}$ solution $(2 \times 40 \mathrm{~mL}$ ). The organic phase was evaporated under reduced pressure and the waxy red-brown $\mathrm{FeOl}_{3}$ dried under vacuum (rotary pump) at $50{ }^{\circ} \mathrm{C}$ (oil bath) for a few hours. To synthesize the NPs, $\mathrm{FeOl}_{3}(1.27 \mathrm{~g}, 1.52 \mathrm{mmol})$ and oleic acid (240 $\left.\mu 1,0.76 \mathrm{mmol}\right)$ were dissolved in trioctylamine $(13 \mathrm{~mL})$ in a $50-\mathrm{mL}$ three-neck round-bottom flask, equipped with condenser and thermometer, under magnetic stirring and $\mathrm{N}_{2}$ atmosphere. The reaction mixture was heated to reflux $\left(\mathrm{ca} .360^{\circ} \mathrm{C}\right)$ at a rate of $10^{\circ} \mathrm{C} / 3 \mathrm{~min}$ and aged for $30 \mathrm{~min}$ using a programmable heating unit. After cooling to RT, the NPs were precipitated from the reaction 
136 mixture by adding acetone $(60 \mathrm{~mL})$, separated by centrifugation, and dispersed in toluene (15 $137 \mathrm{~mL}$ ). To purify the NPs, this procedure was repeated two more times. The iron content of the 138 toluene dispersion, measured by the UV-Vis procedure described below, was $0.68 \mathrm{~g} / \mathrm{L}$. Such 139 low concentration was required to ensure colloidal stability of these NPs.

140

141 Determination of the iron content of NP dispersions

142 ICP-OES procedure. The Fe content in SMA-derived NPs was determined by inductively 143 coupled plasma-optical emission spectroscopy (ICP-OES) (ICAP 6300 Duo, Thermo Fisher 144 Scientific) and an external calibration methodology. The limit of detection (LOD) calculated 145 for iron as $5 \mathrm{ppb}$. For the analysis, the toluene dispersion of SMA-derived NPs $(0.5 \mathrm{~mL})$ was 146 heated in a porcelain crucible over a heating plate and the solvent was evaporated. The solid 147 residue was dissolved in aqua regia $(2 \mathrm{~mL})$, heated until complete evaporation and the solid 148 residue was then dissolved in $0.5 \mathrm{M}$ aqueous $\mathrm{HCl}$. The iron content was then measured by ICP149 OES.

$150 \mathrm{UV}-\mathrm{Vis}$ procedure. This procedure is described in detail in (Mondini et al., 2015) and is here 151 briefly outlined. About $0.1 \mathrm{~mL}$ of toluene NP dispersion is dissolved in aqua regia and 152 evaporated. The residue is re-dissolved in $0.1 \mathrm{M} \mathrm{HCl}$ and the solution is buffered with PBS (pH 153 7). The solution is then treated with tiron in excess to form the red iron complex $\left[\mathrm{Fe}(\text { tiron })_{3}\right]^{3-}$ 154 that is spectrophotometrically determined by a calibration method.

155

$156 \mathrm{PANI} / \mathrm{Fe}_{3} \mathrm{O}_{4} \mathrm{NPs}$ composites preparation using $\mathrm{H}_{2} \mathrm{O}_{2}$ as the oxidant

$157500 \mathrm{mg}$ of $\mathrm{N}$-4-(aminophenyl)aniline (aniline dimer, AD) were dispersed in $30 \mathrm{~mL}$ of water 158 acidified with $2.7 \mathrm{~mL}$ of $\mathrm{HCl} 1 \mathrm{M}(\mathrm{AD} / \mathrm{HCl}=1$, molar ratio $)$. The mixture was stirred for 30 159 minutes. Then, $1.2 \mathrm{~mL}$ of an aqueous of $\mathrm{H}_{2} \mathrm{O}_{2} 35 \%$ was added $\left(\mathrm{H}_{2} \mathrm{O}_{2} / \mathrm{AD}=5\right.$, molar ratio, 160 followed by different amounts of $\mathrm{Fe}_{3} \mathrm{O}_{4}$ NPs. After $24 \mathrm{~h}$, a dark green solid was recovered by 161 filtration, washed with water and acetone abundantly until clearness of the mother liquors and 162 dried in an over at $60^{\circ} \mathrm{C}$ until it reached a constant weight.

163

$164 \mathrm{PANI} / \mathrm{Fe}_{3} \mathrm{O}_{4} \mathrm{NPS}$ composites preparation using $\mathrm{O}_{2}$ as the oxidant

$165500 \mathrm{mg}$ of $N$-4-(aminophenyl)aniline (aniline dimer, AD) were dispersed in $30 \mathrm{~mL}$ of water 166 acidified with $2.7 \mathrm{~mL}$ of $\mathrm{HCl} 1 \mathrm{M}(\mathrm{AD} / \mathrm{HCl}=1$, molar ratio $)$. The mixture was stirred for 30 167 minutes and then different amounts of $\mathrm{Fe}_{3} \mathrm{O}_{4} \mathrm{NPs}$ were added. The reaction mixture was stirred 168 under pressure of molecular oxygen ( 3 bar) for $72 \mathrm{~h}$ at $80^{\circ} \mathrm{C}$. Finally, a dark green solid was 
169 recovered by filtration, washed with water and acetone abundantly until clearness of the mother 170 liquors and dried in an over at $60^{\circ} \mathrm{C}$ until it reached a constant weight.

172 Materials Characterization

173 The TEM images, electron diffraction (ED) patterns, Electron Energy Loss Spectroscopy 174 (EELS) data, and Energy Filtered (EF-TEM) TEM images were recorded by a ZEISS LIBRA $175200 \mathrm{FE}$ TEM that operates at $200 \mathrm{kV}$ and is equipped with a second generation in-column $\Omega$ 176 filter and a HAADF detector for STEM imaging. The samples were prepared by dropping $7 \mu 1$ 177 of NP dispersion on a copper grid coated with a 1-3 nm carbon film and letting it dry overnight.

178 The magnetite NPs were colloidally dispersed in toluene, whereas the composite samples were 179 suspended in isopropanol. TEM and EF-TEM Images were processed by means of the iTEM 180 TEM Imaging Platform software (Olympus) and the NP size distribution was measured by the 181 software PeBBles (Mondini,et al., 2012).

182 FT-IR spectra of composites were recorded in the transmittance mode in the range 500-4000 $183 \mathrm{~cm}^{-1}$ at 64 scans per spectrum and a $2 \mathrm{~cm}^{-1}$ resolution by JASCO FT/IR-410 spectrophotometer 184 (JASCO Corporation, Tokyo, Japan). A small amount of each sample was dispersed in $\mathrm{KBr}$ and 185 compressed in $13 \mathrm{~mm}$ diameter pellets with a 10-ton hydrostatic press for $15 \mathrm{~min}$.

186 X-ray powder diffraction (XRPD) analyses were carried out in a $2 \theta$ range between $10^{\circ}$ and 187 80ºn a Philips PW 3710 Bragg-Brentano goniometer (Philips, Amsterdam, Netherlands) 188 equipped with a scintillation counter, a slit with $1 \circ$ divergence, a receiving slit of $0.2 \mathrm{~mm}$ and a $1890.04^{\circ}$ Soller slit system. A graphite-monochromatic $\mathrm{Cu} \mathrm{K \alpha}$ radiation was adopted at a nominal $190 \mathrm{X}$-ray power of $40 \mathrm{kV} \times 40 \mathrm{~mA}$.

191 The metal loss in the $\mathrm{Fe}_{3} \mathrm{O}_{4} / \mathrm{PANI}$ composites was determined by atomic absorption 192 spectroscopy on a AAnalyst 100 PerkinElmer instrument.

193 Magnetization measurements of composites synthesized using $\mathrm{H}_{2} \mathrm{O}_{2}$ were carried out by a 194 Quantum Design MPMS XL-5 SQUID magnetometer. Weighted amounts of composite were 195 sealed in Teflon tape. Field-cooled (FC) and zero-field-cooled (ZFC) magnetization curves 196 were recorded in the $5-300 \mathrm{~K}$ range. After cooling the sample from 300 to $5 \mathrm{~K}$ in zero field, the 197 ZFC magnetization was recorded on heating to $300 \mathrm{~K}$ using a measuring field $H_{\text {meas }}=10$ Oe. 198 Next, the FC magnetization was measured $\left(H_{\text {meas }}=10\right.$ Oe $)$ while cooling the sample from 300 199 to $5 \mathrm{~K}$ under $H_{\text {cool }}=10 \mathrm{Oe}$. In the case of the sample with the largest NPs (PANI/NP27), the 200 highest temperature was set to $350 \mathrm{~K}$. Magnetization isotherms (hysteresis loops) were recorded 201 between $+50 \mathrm{kOe}$ and $-50 \mathrm{kOe}$ at $5 \mathrm{~K}$ after cooling in zero magnetic field. All data were 202 corrected for the diamagnetism of the sample holder and PANI and then scaled to the NP mass. 
203 The ac magnetic properties were determined using an Agilent 4395A impedance analyzer using 204 a coaxial short 16454A magnetic material test fixture, and a test of the dielectric properties was 205 performed using a parallel plate method with the 16453A dielectric material test fixture.

\section{Results and discussion}

209 Magnetite nanoparticles

210 To study how the NP size affects the catalytic polymerization of AD and the properties of the 211 resulting nanocomposite, we prepared magnetite NPs of different size by different procedures, 212 each yielding NPs with good size dispersity and uniform shape. We used thermal decomposition 213 to prepare medium and large NPs (Hyeon et al., 2001; Park et al., 2004), whereas very small 214 NPs were synthesized by metal vapor synthesis technique (Barbaro et al., 2015; Campisi et al., 215 2019). TEM images of the NP samples are reported in Figure 1. We obtained the NP 216 morphological parameters by analyzing the TEM images using the Pebbles software 217 (Mondini,et al., 2012) and collected them in Table 1 (The histograms of the NP diameter can 218 be found in the ESI) The three NP samples have largely different size: the diameters span more 219 than an order of magnitude (2.3 to $27 \mathrm{~nm}$ ) while the surface (related to the catalytic activity) 220 and the volume (related to the magnetic properties of the composite) span more than two and 221 three orders of magnitude, respectively. The size dispersion is good to excellent. The $28 \%$ 222 dispersion of NP2 may seem large but one should note that the standard deviation of $0.64 \mathrm{~nm}$ 223 is smaller than the magnetite cell size $(0.84 \mathrm{~nm})$. All NPs have spherical shape, except for the 224 largest nanoparticles in NP27 that display cuboidal shape (see ESI for further TEM images of 225 NP27). The crystal phase of the NP27 and NP10 samples was confirmed to be spinel (cubic 226 ferrite) by ED (see ESI). No diffraction ring can be seen in the ED pattern of NP2 NPs. As 227 already reported (Barbaro et al., 2015), such small NPs are crystalline, and the absence of 228 diffraction rings can then be ascribed to the very wide breadth of the rings. Finally, we note that 229 in this paper, for the sake of clarity, we use the term "magnetite" or " $\mathrm{Fe}_{3} \mathrm{O}_{4}$ " NPs to indicate 230 iron oxide NPs with cubic ferrite crystal structure and composition $\mathrm{Fe}_{3-x} \mathrm{O}_{4-x}(0 \leq x \leq 1)$, varying 231 between magnetite and maghemite. 


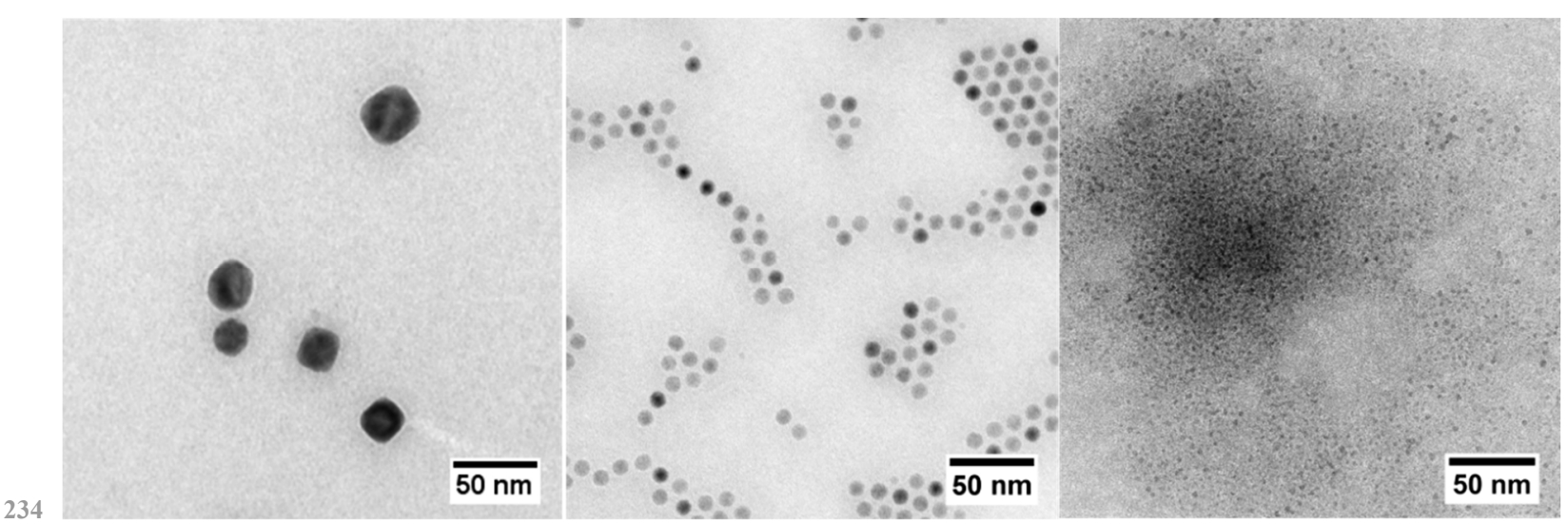

Figure 1. TEM images of as synthesized NPs. a) NP27, b) NP10 c) NP2.

Table 1. Parameters of the distribution of the diameter of as-synthesized magnetite NPs. The number of measured NPs $N$ is reported along with the mean, standard deviation, and variation coefficient of the diameter distribution.

\begin{tabular}{ccccc}
\hline Sample & $N$ & $\begin{array}{c}\text { Mean } \\
(\mathrm{nm})\end{array}$ & $\begin{array}{c}\text { Standard } \\
\text { deviation }(\mathrm{nm})\end{array}$ & $\begin{array}{c}\text { Variation } \\
\text { coefficient }(\%)\end{array}$ \\
\hline NP2 & 463 & 2.3 & 0.64 & 28 \\
NP10 & 1417 & 10.0 & 0.43 & 4.3 \\
NP27 & 181 & 27.1 & 4.3 & 16 \\
\hline
\end{tabular}

242 Polymerization of $\mathrm{AD}$ in the presence of $\mathrm{Fe}_{3} \mathrm{O}_{4}$ nanoparticles

243 The dependence of the catalytic activity of $\mathrm{Fe}_{3} \mathrm{O}_{4}$ NPs on their size was evaluated in the AD 244 oxidative polymerization. The catalytic performance of the NPs is assessed keeping in mind 245 that the NPs are both the catalyst and the magnetic load.

246 In Figure 2 the yield (mass \%) of composites PANI/Fe $\mathrm{O}_{4} \mathrm{NPs}$ is plotted versus the NP/AD 247 molar ratio, where the numerator is the amount of $\mathrm{Fe}_{3} \mathrm{O}_{4}$ contained in the NPs. 
a)

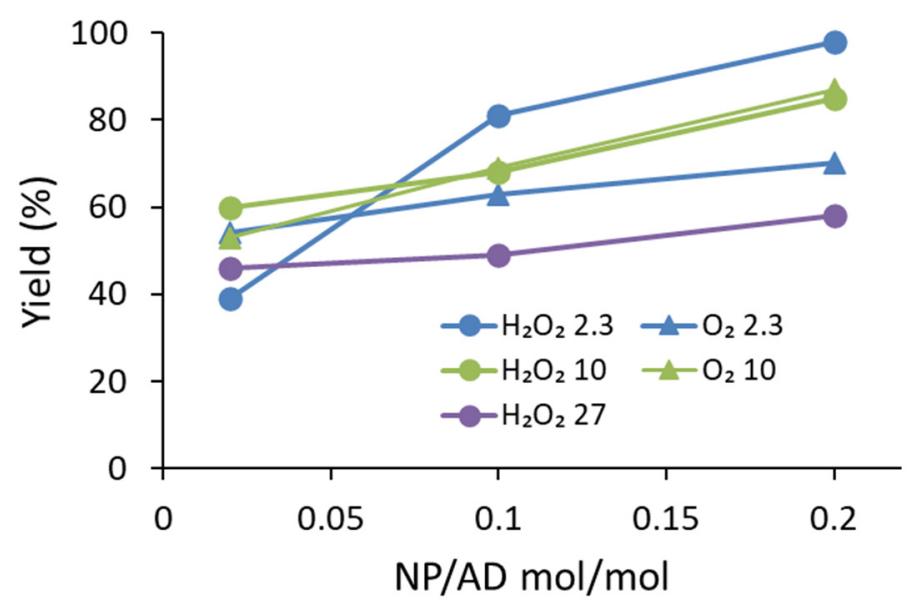

b)

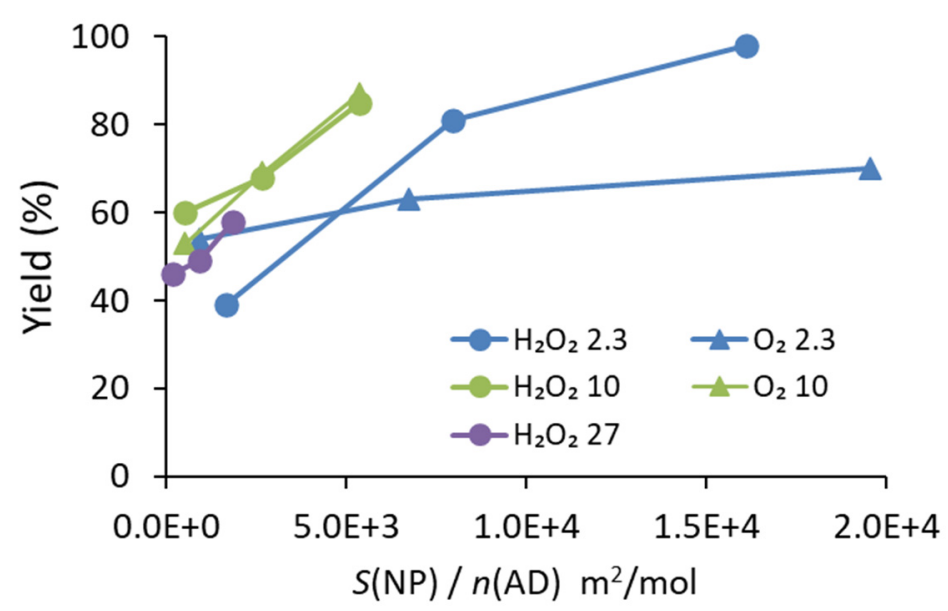

250 Figure 2. Yield (mass \%) of composite from the AD polymerization in the presence of 251 magnetite NPs. The yield is plotted as a function of (a) the NP/AD molar ratio and (b) the ratio 252 of the NP surface area $S$ to the molar amount $n$ of AD.

254 The NP/AD molar ratio was chosen so to explore a NP/AD range that would reasonably yield 255 composites with the desired electrical and magnetic properties. As previously reported, the 256 oxidant used for the polymerization reaction $\left(\mathrm{H}_{2} \mathrm{O}_{2}\right.$ or $\left.\mathrm{O}_{2}\right)$ does not significantly affect the 257 properties of the $\mathrm{PANI} / \mathrm{Fe}_{3} \mathrm{O}_{4}$ composites (Della Pina et al., 2012). Because $\mathrm{H}_{2} \mathrm{O}_{2}$ is especially 258 attractive for the liquid-phase oxidation, due to the high percentage of active oxygen and the 259 production of water as only by-product, we here focused on composites obtained using $\mathrm{H}_{2} \mathrm{O}_{2}$. 260 Polymerizations carried out using $\mathrm{O}_{2}$ as the oxidant are included for comparison.

261 In all experimental conditions explored, the yield increases with the amount of NPs, confirming 262 the catalytic role of the NPs. More insight can be gained when the yield is plotted as a function 263 of the ratio $S / n(\mathrm{AD})$, where $S$ is the total core surface area of the NPs and $n(\mathrm{AD})$ is the molar 264 amount of $\mathrm{AD}$ (Figure 2b). The area $S$ is estimated from the total mass of $\mathrm{Fe}_{3} \mathrm{O}_{4}$ in the composite 
$265 m$ and their mean surface area $\langle s>$ and volume $<v>$ measured from TEM images as $S=m<s>$ $266 / \rho\langle v\rangle$, where $\rho=5.17 \mathrm{~g} / \mathrm{cm}^{3}$ is the density of magnetite. When small NP2 are used, a 267 significant fraction of the NPs is not embedded in the final composite (see ESI). We corrected 268 the data for NP2 in Figure $2 b$ by considering the surface of embedded NPs only. The lack of 269 smallest NPs can be attributed to their very high surface area that makes them more sensitive 270 to the environment. The stability of such small particles is difficult to study and aggregation 271 and dissolution phenomena have to be considered.

272 The yield vs. $S / n(\mathrm{AD})$ data for the larger NPs (NP10 and NP27) is scarcely affected by the NP 273 size or oxidant. They cluster along a straight line, indicating that the slow initial steps of the 274 polymerization (Tzou and Gregory, 1992) occur on the surface of the NP inorganic core. This 275 linear behavior extends to high conversion, suggesting that the NPs are stable against 276 aggregation in the reaction conditions.

277 The yield of NP2 is always lower than that of the larger NPs. When $\mathrm{H}_{2} \mathrm{O}_{2}$ is used as an oxidant, 278 the yield data of NP2 is parallel to that of the larger NPs but shifted to the right (Figure 2b). 279 This suggests that partial NPs aggregation occurs during the polymerization, as confirmed by 280 TEM (see Figure 4 below), thus decreasing the available active sites for the AD oligomerization 281 and hindering transport of $\mathrm{AD}$ to them. When $\mathrm{NP} / \mathrm{AD}=0.2 \mathrm{~mol} / \mathrm{mol}$, parallelism is lost as the 282 yield levels off because most of the AD has undergone polymerization. When NP2 are used 283 with $\mathrm{O}_{2}$, the yield at $\mathrm{NP} / \mathrm{AD}=0.02 \mathrm{~mol} / \mathrm{mol}$ is comparable to that of the larger NPs showing 284 that the NP surface is as active as in the other cases. However, the yield increases very slowly 285 with the $S / n(\mathrm{AD})$ ratio. We admit that we could not find a better explanation for this behavior 286 than invoking extensive NP aggregation when the NP/AD ratio is larger than 0.02 and $\mathrm{O}_{2}$ is 287 used as an oxidant.

288 In summary, magnetite NPs with size from 2.3 to $27 \mathrm{~nm}$ are able to catalyze the polymerization 289 of $\mathrm{AD}$, using either $\mathrm{H}_{2} \mathrm{O}_{2}$ or $\mathrm{O}_{2}$ as oxidant, and with yield ranging from $39 \%$ to $98 \%$. It is 290 therefore feasible to produce magnetic PANI composites with magnetite NPs spanning a size 291 range wider than an order of magnitude.

\section{$293 \mathrm{PANI} / \mathrm{Fe}_{3} \mathrm{O}_{4} \mathrm{NPS}$ characterization}

294 All composites were characterized by different techniques in order to investigate the effect of 295 the presence of the magnetic NPs in the final materials. Here, we report the results obtained for 296 the composites synthesized in the presence of $\mathrm{H}_{2} \mathrm{O}_{2}$ as the oxidant and using a NP/AD molar 297 ratio of 0.2 , because they are the most representative. These three composites are named 298 PANI/NP2, PANI/NP10, and PANI/NP27. 
299 The amount of magnetic NPs inside the composites was indirectly evaluated by the analysis of 300 iron in the mother liquors obtained after the filtration of the materials. The results show that 301 only for the composite PANI/NP2 the content of NPs within the polymeric matrix is not 302 quantitative $\left(16 \%\right.$ of $\mathrm{Fe}_{3} \mathrm{O}_{4}$, cfr. ESI). In all other cases, during the polymerization reaction the 303 inorganic component is completely embedded in the polymeric matrix $\left(33 \%\right.$ of $\mathrm{Fe}_{3} \mathrm{O}_{4}$ in $304 \mathrm{PANI} / \mathrm{NP} 10,31 \%$ of $\mathrm{Fe}_{3} \mathrm{O}_{4}$ in $\left.\mathrm{PANI} / \mathrm{NP} 27\right)$.

305

306 Fourier-transform infrared (FT-IR) spectroscopy

307 All composites comprise a polymeric matrix consisting of PANI in the form of conducting 308 emeraldine, as shown by characteristic infrared bands (see ESI). In particular, the broad band 309 between $3400-1800 \mathrm{~cm}^{-1}$ confirms that the polymer was obtained in highly conjugated form 310 (Šeděnková et al., 2008), the band at 1570 and $1490 \mathrm{~cm}^{-1}$ are assigned to the $\mathrm{C}=\mathrm{C}$ stretching 311 vibration of quinoid and benzenoid rings respectively, whereas $\mathrm{C}-\mathrm{N}$ stretching vibration is 312 responsible of the band at $1304 \mathrm{~cm}^{-1}$ (Cionti et al., 2020). The band at $1240 \mathrm{~cm}^{-1}$ is due to C$313 \mathrm{~N}^{+}$s stretching vibration (Šeděnková et al., 2008) and that to $1146 \mathrm{~cm}^{-1}$ derives from $\mathrm{Q}=\mathrm{NH}^{+}$314 B, B-NH ${ }^{+}-\mathrm{B}$ stretching (Mišurović et al., 2019). C-H out-of-plane bending vibrations of 1,4315 disubstituted aromatic rings lead to the bands in the $820-800 \mathrm{~cm}^{-1}$ range (Socrates, 2004). 316 Finally, the two bands at 800 and $759 \mathrm{~cm}^{-1}$ suggest the presence of C-H vibrations of 1,2,4317 trisubstituted and 1,2-disubstituted rings (Socrates, 2004).

319 X-ray powder diffraction (XRPD)

320 The XRPD patterns of PANI/Fe $\mathrm{O}_{4} \mathrm{NPs}$ composites are reported in Figure 3.

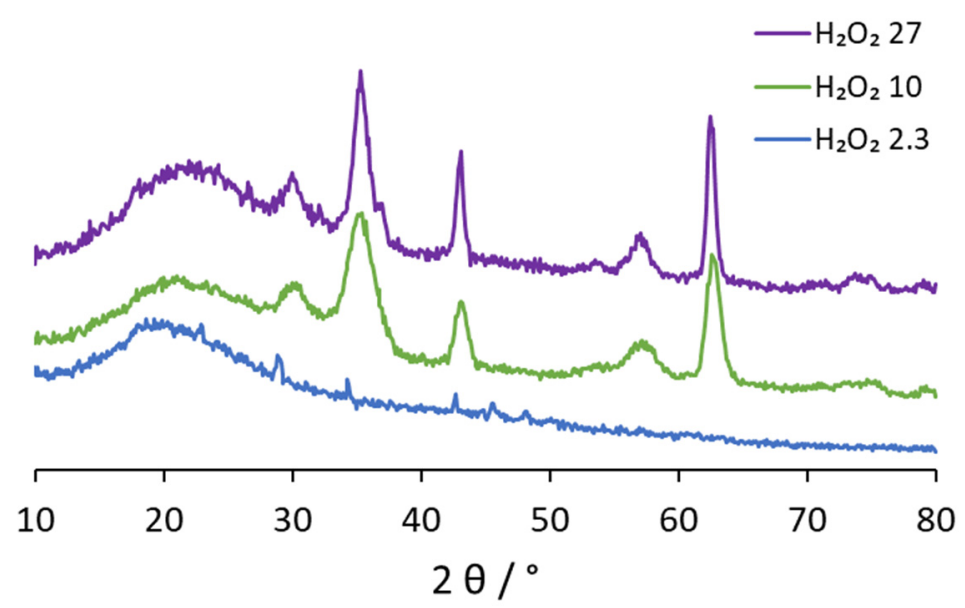


Figure 3. XRPD patterns of $\mathrm{PANI} / \mathrm{Fe}_{3} \mathrm{O}_{4} \mathrm{NPs}$ composites.

326 The XRPD patterns of the composites show the typical Bragg diffraction peak at $2 \theta \approx 20^{\circ}$ for 327 the polymer. Peaks at $2 \theta=30.1,35.6,43.2,53.8,57.2$, and $63.0^{\circ}$ are prominent in the patterns 328 of composites including NP10 and NP27. They can be assigned to cubic ferrite (spinel) NPs, 329 showing that during the synthesis of the composites the magnetic NPs were embedded into the 330 polymer. The XRPD pattern of PANI/NP2 composites only shows the peak of PANI. Since the 331 presence of small NP2 in the composite is established by TEM techniques (see the net section), 332 the absence of the peaks typical of the spinel structure could be due to the amorphousness of 333 the NPs. However, NP2 were already shown to be crystalline (Barbaro et al., 2015). Therefore, 334 the inability to detect the spinel peaks is ascribed to combined effect of the larger peak width 335 expected for smaller NPs (about fourfold wider than for PANI/NP10) and the lower magnetite 336 content in PANI/NP2.

337

338 TEM microscopy

339 The TEM investigation of the composites has two goals: to establish the presence of magnetite 340 NPs within the composites and to ascertain whether the NPs underwent morphological changes 341 during the polymerization reaction. The investigation could not rely on conventional TEM 342 imaging only because the composite granules are thick enough to be opaque to the electron 343 beam except for the very edges of the granules (see ESI). 
a)

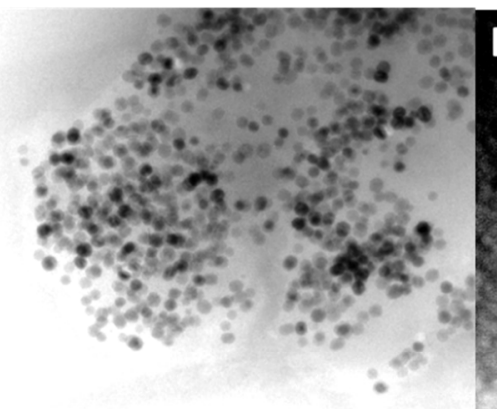

b)

$100 \mathrm{~nm}$

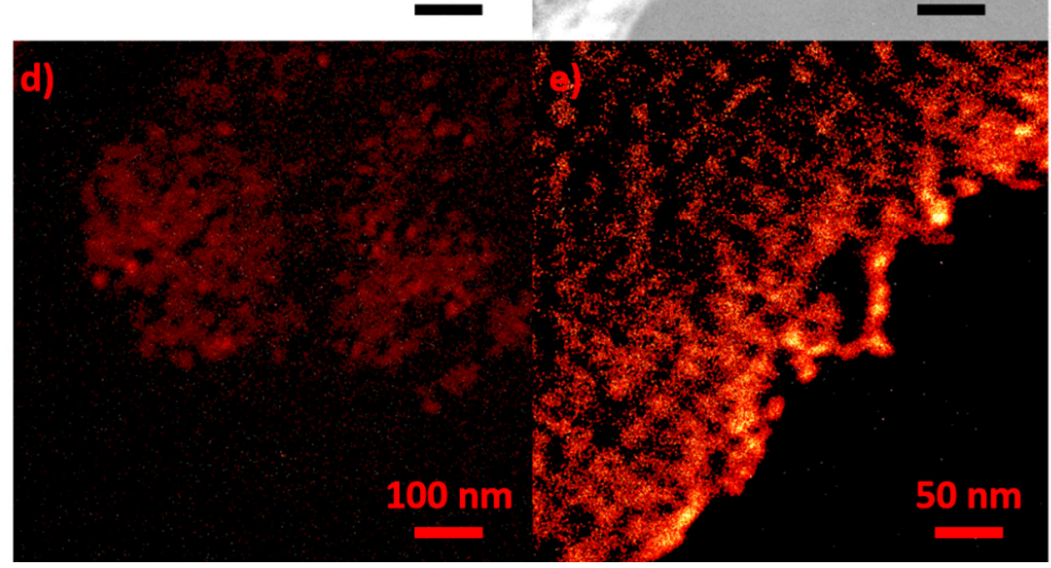

c)
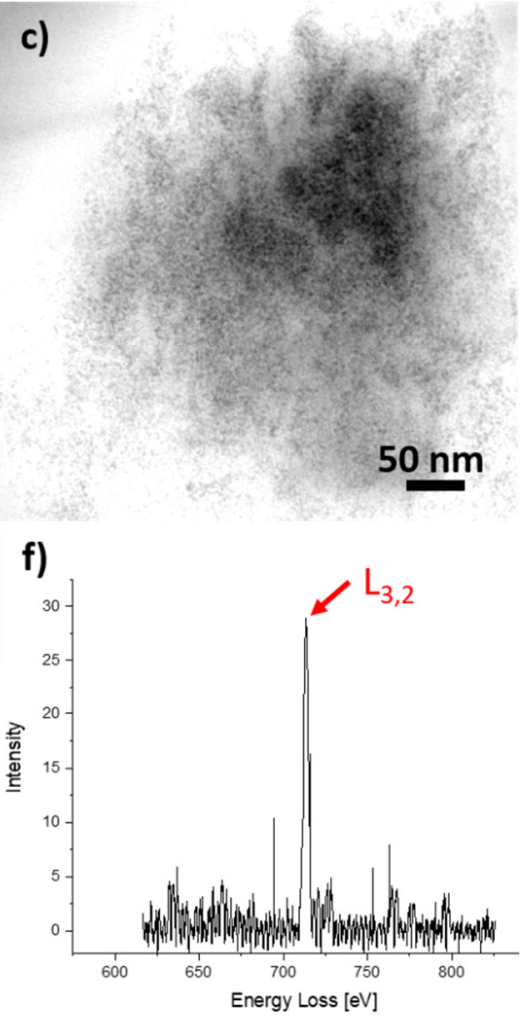

346 Figure 4. TEM of PANI/Fe $\mathrm{O}_{4} \mathrm{NPs}$ composites. EF-TEM images filtered at $30 \mathrm{eV}$ of a) 347 PANI/NP27, b) PANI/NP10, and c) PANI/NP2. False-color EF-TEM images filtered at $713 \mathrm{eV}$ 348 providing Fe maps of d) PANI/NP27 and e) PANI/NP10. The sample regions imaged are the 349 same as in a) and b). f) Background-subtracted EELS spectrum of PANI/NP2 showing the iron $350 L_{3,2}$ peak.

351

352 We thus resorted to energy-filtered TEM (EF-TEM) techniques by which the image is formed 353 by detecting electrons that lost energy during the interaction with sample (Figure 4). In images 354 filtered at $30 \mathrm{eV}$ loss, the contrast between NPs and polymer is enhanced since electrons can 355 lose $30 \mathrm{eV}$ only by interacting with nearly-free aromatic $\pi$ electrons, the so-called plasmonic 356 mode, so that the polymer matrix appears light gray whereas the NPs appear dark. The enhanced 357 contrast between NPs and polyaniline allowed us to identify the NPs inside the composite 358 (Figure 4a-c). The NPs are well dispersed within the composites. Some NP agglomeration was 359 observed in the case of the PANI/NP2 (dark region in Figure 4c). NP measurement was 360 unfortunately reliable only for PANI/NP10 and PANI/NP27. The results are collected in Table 361 2. Comparing this data with those in Table 1, one can see that the NP10 are unchanged while 362 the NP27 decreased in size by about $2 \mathrm{~nm}$ during the polymerization. In both cases, the size 
382 In Figure 5, SEM images of PANI/Fe $\mathrm{O}_{4} \mathrm{NPs}$ composites are displayed.

Table 2. Parameters of the distribution of the diameter of magnetite NPs present in mean, standard deviation, and variation coefficient of the diameter distribution.

\begin{tabular}{ccccc}
\hline Composite & $N$ & $\begin{array}{c}\text { Mean } \\
(\mathrm{nm})\end{array}$ & $\begin{array}{c}\text { Standard } \\
\text { deviation }(\mathrm{nm})\end{array}$ & $\begin{array}{c}\text { Variation } \\
\text { coefficient (\%) }\end{array}$ \\
\hline PANI/NP10 & 335 & 10.2 & 0.63 & $6.2 \%$ \\
PANI/NP27 & 207 & 25.0 & 4.6 & $18 \%$ \\
\hline
\end{tabular}
NPs. PANI/NP2 gave no detectable diffraction rings.

\section{SEM microscopy} $\mathrm{PANI} / \mathrm{Fe}_{3} \mathrm{O}_{4} \mathrm{NPs}$ composites. The number of measured NPs $N$ is reported along with the

We also confirmed the presence of iron in the embedded NPs detecting electrons with energy loss of $713 \mathrm{eV}$, typical of iron ( $L_{2,3}$ peak). The EF-TEM iron maps of PANI/NP27 and PANI/NP10 (Figure 4d,e) show that the iron signal coincides with the NPs as seen in the corresponding $30 \mathrm{eV}$ loss images (Figure 4a,b). In the case of PANI/NP2, the intensity of the EF-TEM iron map was too low to reliably identify the NPs within the composite. The presence of iron in PANI/NP2 was established thanks to the EELS spectrum that displays the iron $L_{3,2}$, peak at $713 \mathrm{eV}$ (Figure 4f). The crystal structure of the embedded NPs was analyzed by ED (see ESI): the ED patterns confirmed that PANI/NP27 and PANI/NP10 comprise cubic ferrite 

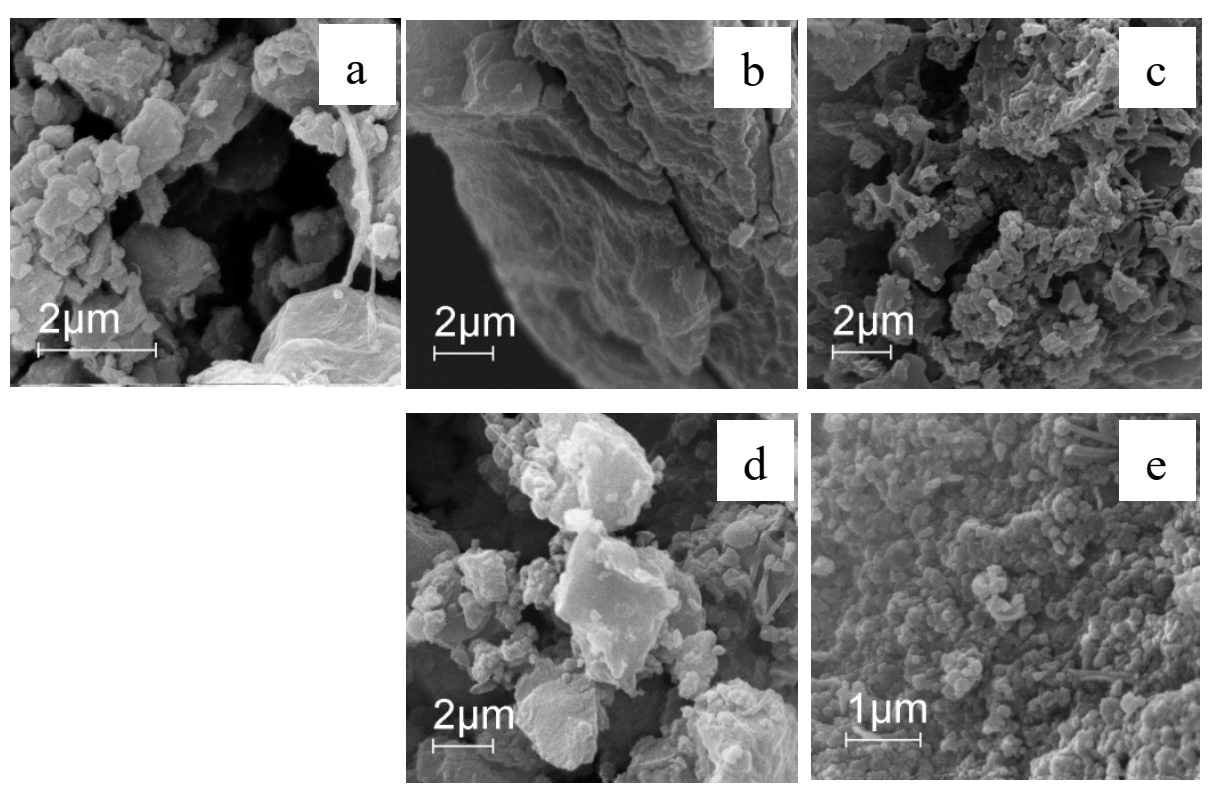

Figure 5. SEM images of $\mathrm{PANI} / \mathrm{Fe}_{3} \mathrm{O}_{4} \mathrm{NPs}$ composites (a: PANI/NP27, b: PANI/NP10, c: 385 PANI/NP2) or $\mathrm{O}_{2}$ as oxidant (d: PANI/NP10, e: PANI/NP2).

387 When compared to PANI obtained by the oxidative polymerization of AD in the presence of a 388 homogeneous catalyst, e.g., Fe(III) (Della Pina et al., 2018), PANI/Fe $\mathrm{O}_{4} \mathrm{NPs}$ composites 389 display a more globular morphology, sparsely intermixed with nanorods. As previously 390 reported (Della Pina et al., 2012), when AD hydrochloride is polymerized by $\mathrm{H}_{2} \mathrm{O}_{2}$ in water in 391 the presence of magnetite NPs dispersed in an organic phase as the catalyst, an interfacial 392 polymerization occurs. Thanks to its solubility in organic solvents, AD hydrochloride can 393 diffuse into the organic phase where $\mathrm{Fe}_{3} \mathrm{O}_{4} \mathrm{NPs}$ are present, and the oxidative polymerization 394 can take place at the interface between the organic and aqueous phases (Scheme 1). 


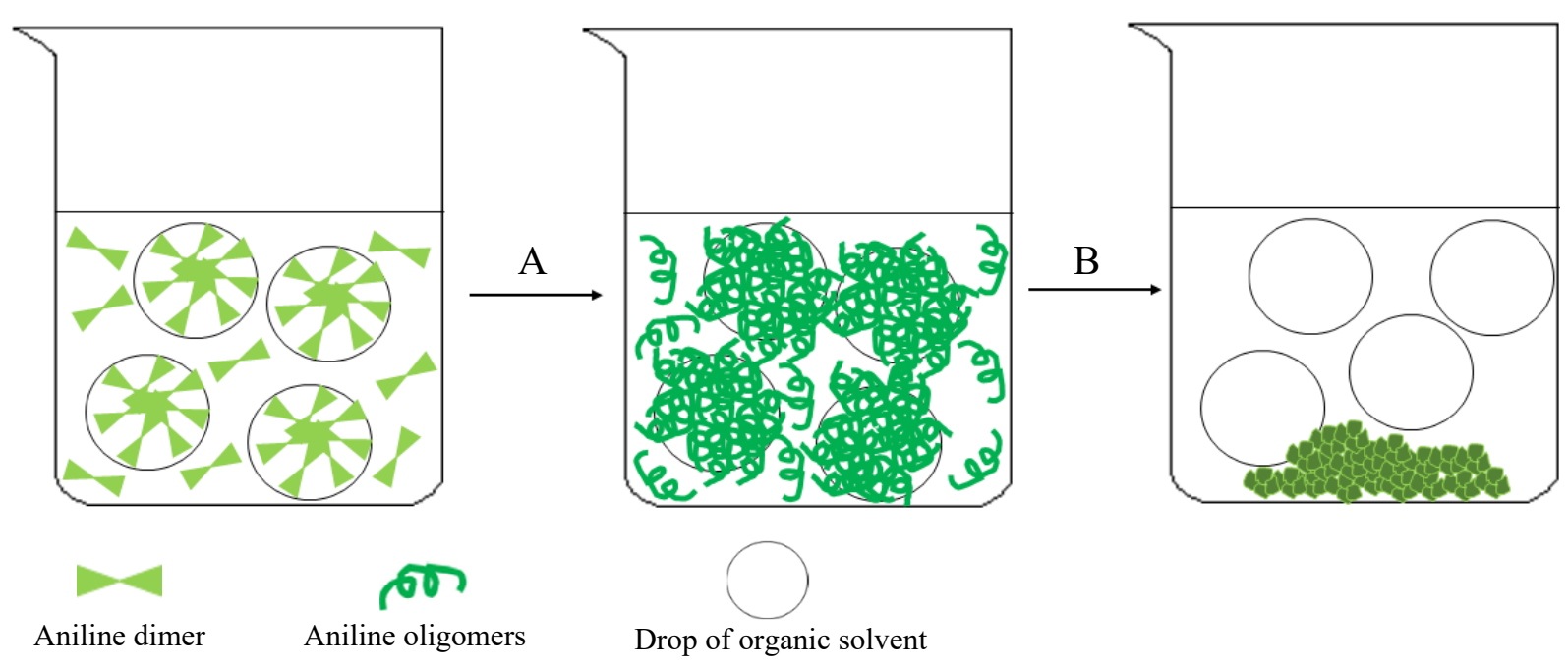

Scheme 1. Proposed mechanism of nanoglobules formation. A) oligomerization step, B) 400 polymerization step.

401

402 During the first steps of the reaction, only PANI oligomers are produced that are soluble in 403 organic solvents and, as a consequence, they can diffuse inside the toluene drops. When the 404 polymeric chains grow longer, they become insoluble and precipitate from the reaction mixture 405 leading to globular materials.

406 Even though this hypothesis of mechanism seems to be in contrast with that previously reported 407 (Della Pina et al., 2012), it has to be considered that the amount of organic phase was changed 408 and the catalysts were obtained by a different synthetic approach (co-precipitation reaction in 409 Della Pina et al., 2012, thermal decomposition here).

410

\section{Magnetic properties}

412 The thermal behavior of the magnetization $M$ of the PANI $/ \mathrm{Fe}_{3} \mathrm{O}_{4} \mathrm{NPs}$ composites (oxidant: $413 \mathrm{H}_{2} \mathrm{O}_{2} ; \mathrm{NP} / \mathrm{AD}=0.02 \mathrm{~mol} / \mathrm{mol}$ ) was investigated by means of $\mathrm{ZFC} / \mathrm{FC}$ experiments (Figure 6). 414 The main magnetic parameters are collected in Table 3. The ZFC/FC curves of the composites 415 are widely different. Reversible behavior is indicated by the identity of the ZFC and FC data, 416 which is clearly visible in the high temperature range for all composites. The reversibility 417 corresponds to the superparamagnetic (SPM) regime where thermal agitation quickly flips the 418 NP magnetization. Upon cooling the two curves diverge as soon as thermal agitation is not able 419 to overcome the barrier for magnetization reversal of some NPs and irreversibility sets in. The 420 irreversibility temperature $T_{\text {irr, }}$, defined as the temperature at which the difference between the $421 \mathrm{FC}$ and the $\mathrm{ZFC}$ magnetization $\left(M_{\mathrm{FC}}-M_{\mathrm{ZFC}}\right) / M_{\mathrm{FC}}=3 \%$, is larger than $\mathrm{RT}$ for PANI/NP27, as 422 low as $110 \mathrm{~K}$ for PANI/NP10, and probably less than $80 \mathrm{~K}$ for PANI/NP2 (in the latter case, 
423 the smallness of $M_{\mathrm{FC}}-M_{\mathrm{ZFC}}$ prevents an accurate determination of $\left.T_{\text {irr }}\right)$. Thus, PANI/NP27

424 contains NPs both in the SPM and blocked regime even at RT, in agreement with the ideal RT

425 blocking diameter of magnetite (26 nm) (Coey, 2010).

${ }_{22}$ Table 3. Magnetic parameters of the $\mathrm{ZFC} / \mathrm{FC}$ magnetization of $\mathrm{PANI} / \mathrm{Fe}_{3} \mathrm{O}_{4} \mathrm{NPs}$ composites.

\section{HWHH}

\begin{tabular}{lcccc} 
Composite & $T_{\text {irr }}(\mathrm{K})$ & $T_{\text {der }}(\mathrm{K})$ & $(\mathrm{K})$ & $<T_{\mathrm{b}}>(\mathrm{K})$ \\
\hline PANI/NP2 & $<80 \mathrm{~K}$ & - & - & - \\
PANI/NP10 & 110 & 60 & 29 & 54 \\
PANI/NP27 & 310 & 250 & 41 & 290 \\
\hline
\end{tabular}
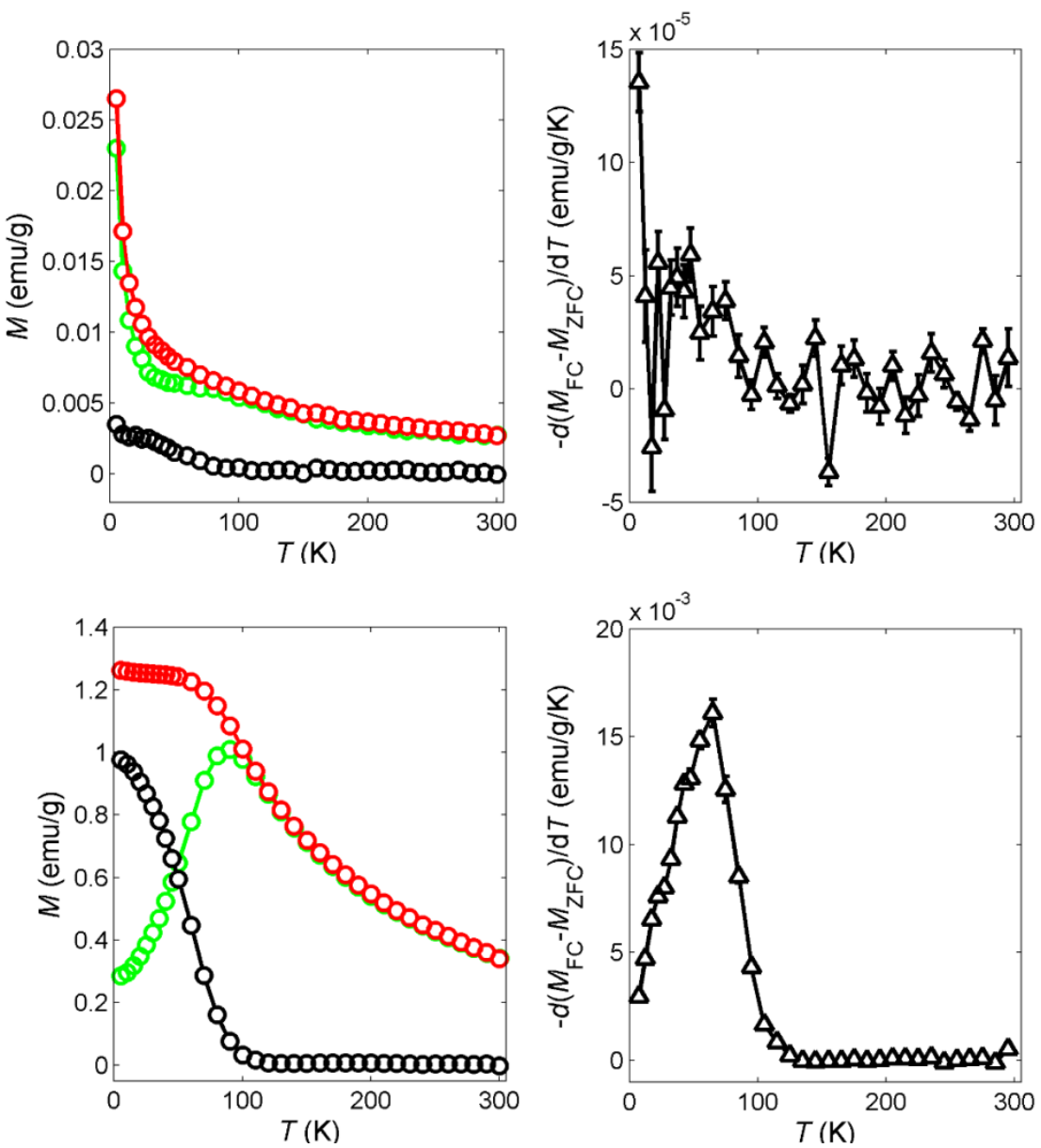

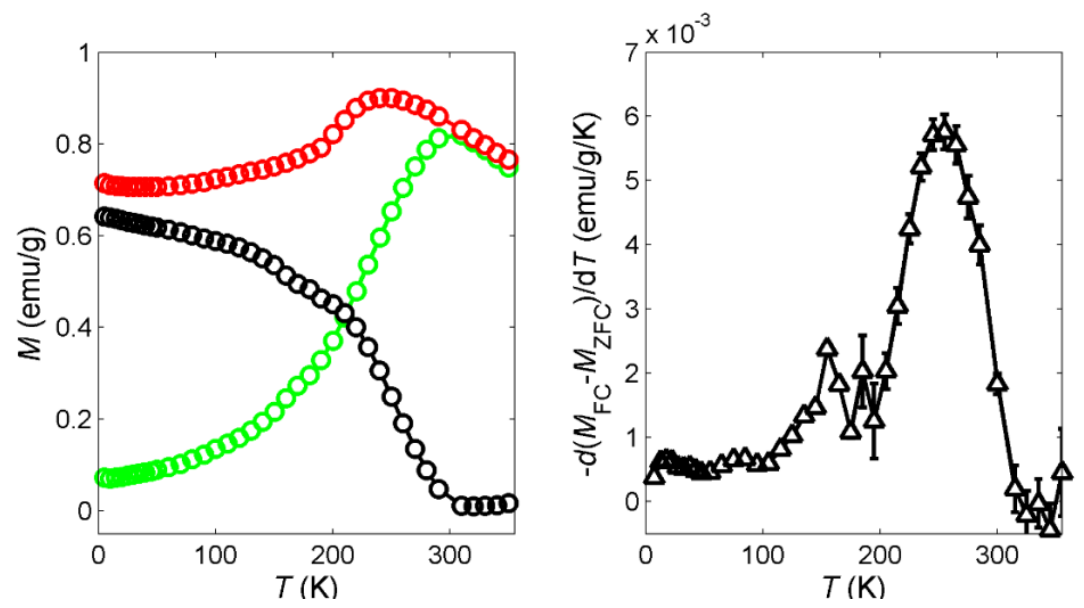

430 Figure 6. Thermal behavior of the low-field $(H=10 \mathrm{Oe})$ magnetization of PANI/Fe $3 \mathrm{O}_{4} \mathrm{NPs}_{\mathrm{S}}$ 431 composites. In the left panels, the ZFC (green) and FC (red) magnetizations are shown along 432 with their difference (black). In the right panels, the $-\mathrm{d}\left(M_{\mathrm{FC}}-M_{\mathrm{ZFC}}\right) / \mathrm{d} T$ derivative is plotted to 433 represent the distribution of the blocking temperatures. Top: PANI/NP2, middle: PANI/NP10, 434 bottom: PANI/NP27. The error bars represent 1 standard deviation; the error bars are not visible 435 when the standard deviation is smaller than the symbol size.

436

437 Upon further cooling below $T_{\text {irr, }}$ more NPs enter the blocked regime. In this temperature region, 438 the three samples display largely different behavior. MFC and MZFC of PANI/NP2 are similar 439 down to $5 \mathrm{~K}$, showing that the NPs in this composite are subject to low barriers for 440 magnetization rotation, as expected for very small NPs. PANI/NP10 displays a strong decrease 441 of $M_{\mathrm{ZFC}}$ and a flattening of $M_{\mathrm{FC}}$ at low temperature. The larger difference between $M_{\mathrm{FC}}$ and ${ }_{442} M_{\mathrm{ZFC}}$ corresponds to larger barriers. The $M_{\mathrm{FC}}$ and $M_{\mathrm{ZFC}}$ of PANI/NP10 and PANI/NP2 are 443 typical of weakly interacting NPs. In the case of PANI/NP27, both $M_{\mathrm{FC}}$ and $M_{\mathrm{ZFC}}$ decrease from $444250 \mathrm{~K}$ down to $5 \mathrm{~K}$, showing strong interparticle interactions and the onset of a frozen spin445 glass-like state where the magnetization is frozen in one of the many minima of a complex free446 energy landscape created by the magnetostatic interaction among the NPs. The presence and 447 freezing temperature of the spin-glass-like state agree with an estimate of the relative strength 448 of the magnetostatic interaction among the NPs (see ESI).

449 A representation of the magnetization rotation barriers of a composite (in terms of blocking 450 temperature $T \mathrm{~b}$ ) can be obtained as the derivative of the difference between the FC and ZFC 451 magnetization $-\mathrm{d}\left(M_{\mathrm{FC}}-M_{\mathrm{ZFC}}\right) / \mathrm{d} T$ (Figure 6, right panels) (Bruvera et al,, 2015). This 452 representation faithfully displays the distribution of $T_{\mathrm{b}}$ in the case of weakly-interacting NPs. 453 When a spin-glass-like state sets in, the concept of $T_{\mathrm{b}}$ of individual NPs is not appropriate but $454-\mathrm{d}\left(M_{\mathrm{FC}}-M_{\mathrm{ZFC}}\right) / \mathrm{d} T$ however provides useful information about the barriers, (Woińska et al, 
4552013 ) though they are a property of the whole sample and cannot be tied to individual NPs. The 456 mode $T_{\text {der }}$ and half-width-half-height $(\mathrm{HWHH})$ of the $T_{\mathrm{b}}$ distribution can be found in Table 3 457 along with the mean barrier $<T_{\mathrm{b}}>$. PANI/NP2 has barriers with $T_{\mathrm{b}}<80 \mathrm{~K}$ but $T_{\text {der }}$ and $<T_{\mathrm{b}}>$ 458 could not be reliably determined. These barriers, which are related to a small part of the total 459 magnetization, as shown by the $M_{\mathrm{FC}}-M_{\mathrm{ZFC}}$ difference, can be attributed to a few NP aggregates. 460 The barriers in PANI/NP10 are distributed about $T_{\text {der }}=60 \mathrm{~K}\left(<T_{\mathrm{b}}>=54 \mathrm{~K}\right)$ and extend down 461 from $100 \mathrm{~K}$ (HWHH $29 \mathrm{~K})$. A similar peak is observed in the PANI/NP27 Tb distribution, but 462 it occurs at higher $T_{\text {der }}=250 \mathrm{~K}\left(<T_{\mathrm{b}}>=290 \mathrm{~K}\right)$ with $\mathrm{HWHH}=41 \mathrm{~K}$. The barrier distribution 463 has a broad tail extending down to $5 \mathrm{~K}$, which is related to collective barriers in the frozen state. 464 The $T_{\mathrm{b}}$ of our composites agree with the $T_{\mathrm{b}}$ measured for magnetite NPs in the $d=10-20 \mathrm{~nm}$ 465 range (Yun et al., 2014) (see ESI for a detailed comparison).

466 To gain more insight into the magnetic properties of the composites, magnetization isotherms 467 were measured between -50 and $+50 \mathrm{kOe}$ at $5 \mathrm{~K}$. The shape of these hysteresis loops (see ESI) 468 and the magnetic parameters collected in Table 4 confirm the almost complete magnetic 469 reversibility of PANI/NP2 at low temperature and the irreversible behavior of PANI/NP10 and PANI/NP27.

In summary, these composite materials display a variety of magnetic behavior that can be selected by choosing the NP size, e.g., the SPM regime can be shifted from RT to $T \approx 5 \mathrm{~K}$.

Table 4. Magnetic parameters from the magnetization isotherm of $\mathrm{PANI} / \mathrm{Fe}_{3} \mathrm{O}_{4} \mathrm{NPs}$ composites. The isotherms were measured between -50 and $+50 \mathrm{kOe}$ at $5 \mathrm{~K}$.

\begin{tabular}{ccccc}
\hline Composite & $M_{50}(\mathrm{emu} / \mathrm{g})^{\mathrm{a}, \mathrm{b}}$ & $M_{\mathrm{rem}}(\mathrm{emu} / \mathrm{g})^{\mathrm{a}}$ & $M_{\mathrm{rem}} / M_{50}$ & $H_{\mathrm{c}}(\mathrm{kOe})$ \\
\hline PANI/NP2 & $55.2 \pm 0.8$ & - & - & - \\
PANI/NP10 & $53.4 \pm 0.7$ & $12.8 \pm 0.2$ & $0.24 \pm 0.02$ & 0.45 \\
PANI/NP27 & $42 \pm 1$ & $6.2 \pm 2$ & $0.15 \pm 0.04$ & 0.61
\end{tabular}

${ }^{\mathrm{a}}$ Mass magnetization referred to the NP mass. ${ }^{\mathrm{b}}$ The magnetization at $50 \mathrm{kOe}$ $\left(M_{50}\right)$ is far from the saturation value.

480 Dynamic electromagnetic absorption and Ferromagnetic resonance

481 Magnetic materials subjected to an external applied field may show losses attributed to different 482 phenomena, also frequency dependent. In quasi static conditions, losses are dominated by the 483 hysteresis loss associated to the reversal of the magnetization direction which occurs whenever 484 a portion of a domain wall moves between different pinning sites corresponding to two local 
485 energy minima and some energy is dissipated to the crystal lattice. Dynamic losses due to the

486 flow of eddy currents and joule heating within the material under ac excitations are associated 487 to the magnetic flux variations. At higher frequencies, when flux penetration may be reduced 488 due to the skin effect, energy dissipation is caused by ferromagnetic resonance, occurring 489 whenever the frequency of the external ac magnetic field coincides to the Larmor precession 490 frequency of the magnetization vector. These effects tend to limit the useful frequency range of 491 magnetic materials in practical devices.

492 Due to their extremely small size, nanosized objects such as NPs do not exhibit large hysteresis 493 or dynamic losses and can thus be suitable as inductors even above the $\mathrm{MHz}$ frequency range. 494 In fact, NPs with diameters of the order of $10 \mathrm{~nm}$ are single domain and magnetization reversals 495 occur only through rotations, which produce very small dissipation with respect to hysteresis.

496 Dynamic losses due to large scale eddy currents are also suppressed in NPs, because inter497 particle conduction is hindered by the organic shell and losses arise at frequencies above $1 \mathrm{GHz}$ 498 only due to the resonant magnetic behavior, and to verify this hypothesis dielectric 499 characterization was also performed up to the $\mathrm{GHz}$ range.

500 The real and imaginary part of the magnetic permeability using the following relations (Fiorillo, 2010):

502

$$
\mu^{\prime}=1+\frac{\operatorname{Im}\left\{\Delta Z_{i n}^{*}\right\}}{f \mu_{0} h \ln \frac{R_{m}}{r_{m}}}
$$

504

$$
\mu^{\prime \prime}=\frac{R e\left\{\Delta Z_{i n}^{*}\right\}}{f \mu_{0} h \ln \frac{R_{m}}{r_{m}}}
$$

507 where $h, R_{\mathrm{m}}$ and $r_{\mathrm{m}}$ are the thickness, the outside diameter, and the inside diameter of the ring 508 sample, respectively, $\Delta Z_{i n}^{*}=Z_{i n, s a m p l e}^{*}-Z_{i n, \text { air }}^{*}$, where $Z_{i n, \text { sample }}^{*}$ and $Z_{\text {in, air }}^{*}$ are the circuit 509 impedances in the the presence or absence of the toroidal sample and $f$ is the frequency. 510 Capacitance and dielectric properties were determined directly and calibrated through a Teflon 511 reference, and show that the dielectric properties are constant across the spectrum observed up 512 to above $1 \mathrm{GHz}$, without any visible resonant absorption (Figure 7). 


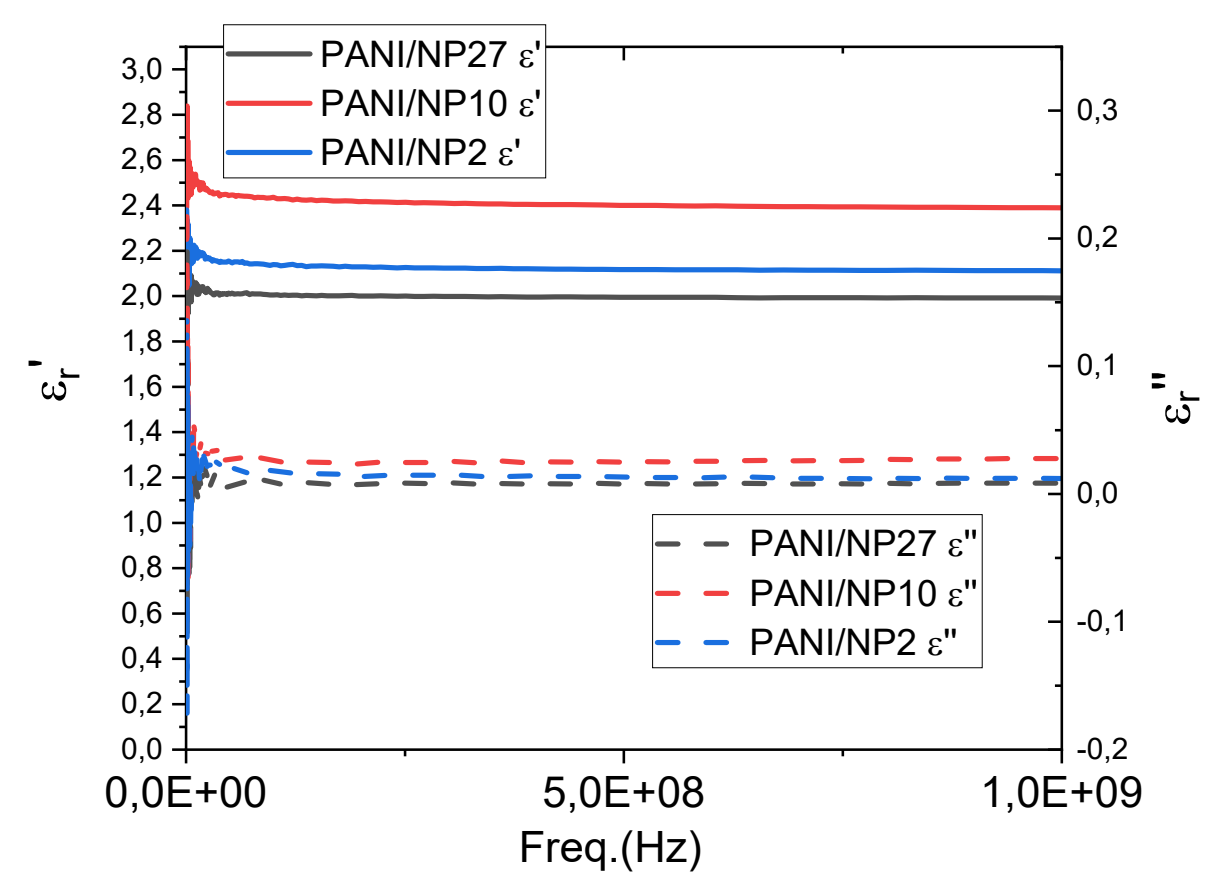

514

Figure 7. Real ( $\left.\varepsilon_{r}^{\prime}\right)$ and imaginary ( $\left.\varepsilon_{r}{ }^{\prime \prime}\right)$ part of the relative permittivity of the PANI/Fe ${ }_{3} \mathrm{O}_{4} \mathrm{NPs}$ 516 composites. The data exhibits a rather flat behavior up to $1 \mathrm{GHz}$.

518 The ac permeability results (Figure 8 ) show that PANI/NP2, the composite with $2.3 \mathrm{~nm}$ single 519 domain NPs, with vanishing $M_{\text {rem }}$ (Table 4) and higher static initial permeability, has the highest 520 real part of permeability also in ac. The other samples show relative permeabilities just slightly 521 above 1. All composites display a wide frequency peak of the imaginary part of the 522 permeability, which starts below $1 \mathrm{GHz}$ and has a peak at 1.1-1.2 GHz, depending on the 523 composite, clearly showing that ac losses are only associated to the ferromagnetic resonance. 524 The large peak width reflects the distribution of particle dimensions and magnetic properties 525 (also due to exact size, chemical composition, etc.). These results, while compatible with 526 previous literature (Yun et al., 2014), extend the experimental frequency range explored and 527 prove that PANI composites with very small $\mathrm{Fe}$ oxide particles are instrumental to the 528 successful use of these materials as energy absorbers in the $\mathrm{GHz}$ range. 


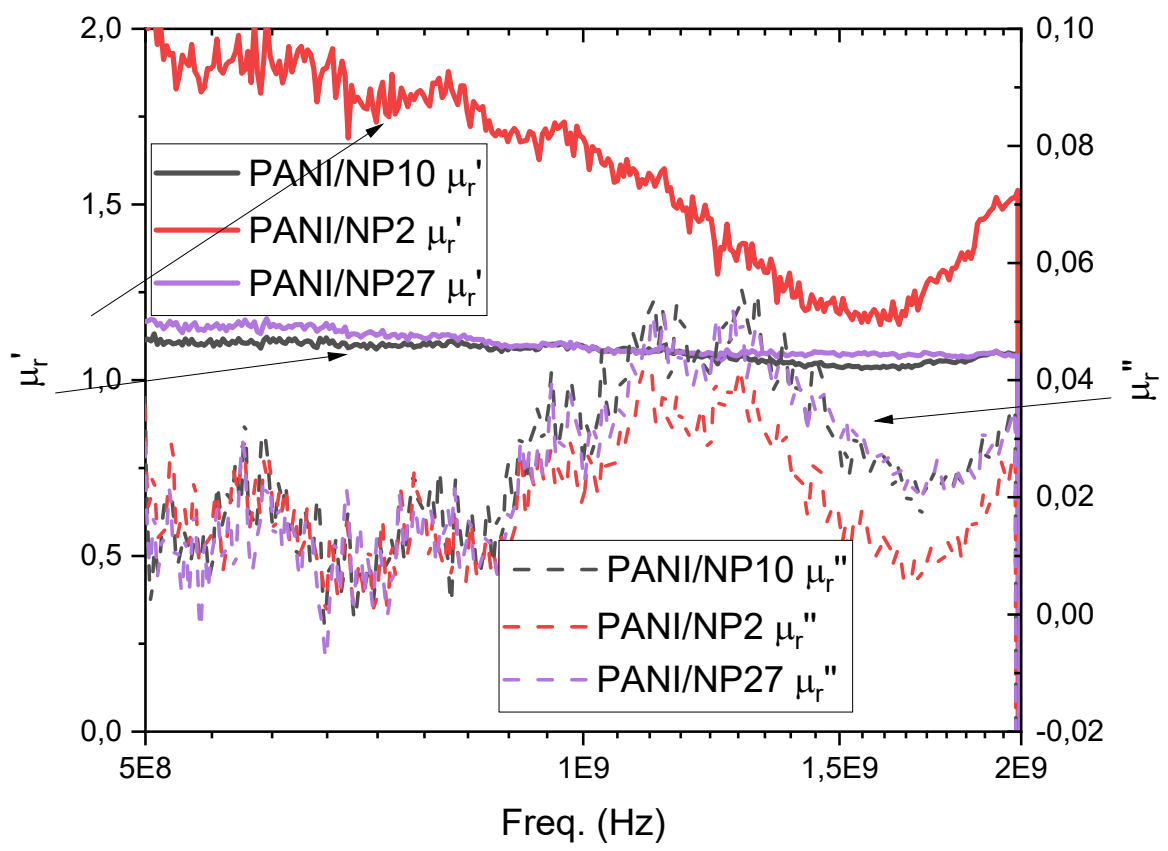

530

Figure 8. Real ( $\left.\mu_{r}{ }^{\prime}\right)$ and imaginary ( $\mu_{r}$ ") part of the relative permeability of the samples. $\mu_{r}$ " shows an absorption behavior, due to the ferromagnetic resonance, at frequencies slightly above $5331 \mathrm{GHz}$.

\section{Conclusions}

537 We have shown that magnetite NPs spanning the size range from 2.3 to $27 \mathrm{~nm}$ are able to 538 catalyze the oxidative polymerization of the aniline dimer and remain within the polymer so to 539 form $\mathrm{PANI} / \mathrm{Fe}_{3} \mathrm{O}_{4}$ composites. The yields are in all cases high enough to be of practical 540 importance. Their dependence on the NP surface area shows that i) the NPs are stable against 541 aggregation in the reaction conditions, ii) the catalytic effect is due to the NPs (and not to $\mathrm{Fe}^{3+}$ 542 ions leached into the aqueous phase), and iii) the NPs are a main factor affecting the 543 polymerization yield. The smallest NPs seem to be not completely stable in the reaction 544 conditions probably because of their very high surface area that makes them more sensitive to 545 surface effects such as aggregation and degradation. The embedded NPs retain the shape and 546 size of the pristine NPs and are well-dispersed in the PANI matrix.

547 The NPs in the composite do not interact by direct exchange, i.e., they retain their magnetic 548 individuality thanks to the oleic acid coating that prevents direct contact between the NPs. This 549 enables easy modulation of the composite magnetic properties based on the NP size. Occurrence 550 of NP direct contact in the composite would have led to unpredictable magnetic properties 
571 References

depending on the details of the polymerization. For the dynamic electromagnetic behavior, the imaginary part of the permeability only shows an absorption, not associated with any dielectric loss, but rather due to the ferromagnetic resonance, at frequencies slightly above $1 \mathrm{GHz}$. Our synthetic method thus provides a pathway to prepare composites with tailored magnetic properties by customizing the size of the magnetite NPs. It would also be conceivable to prepare composites containing NPs with different size in controlled proportion.

Funding. Financial support from Fondazione Cariplo (Milano, Italy) under Grant no. 20120872 (Magnetic-nanoparticle-filled conductive polymer composites for EMI reduction) is gratefully acknowledged.

Conflict of interest. The authors declare no conflict of interest.

\section{Supplementary data}

Electronic Supplementary Material associated with this article can be found in the online

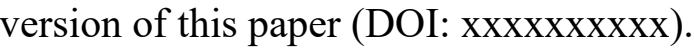

Ahmadkhani L, Mostafavi E, Ghasemali S, Baghban R, Pazoki-Toroudi H, Davaran S, Malakootikhah J, Asadi N, Mammadova L, Saghfi S, Webster TJ, Akbarzadeh A (2019) Development and characterization of a novel conductive polyaniline-gpolystyrene $/ \mathrm{Fe}_{3} \mathrm{O}_{4}$ nanocomposite for the treatment of cancer. Artif Cells Nanomed Biotechnol 47(1):873-881. doi: 10.1080/21691401.2019.1575839

Bhadra S, Khastgir D, Singha NK, Lee JH (2009) Progress in preparation, processing and applications of polyaniline. Prog Polym Sci 34:783-810. doi: 10.1016/j.progpolymsci.2009.04.003

Barbaro D, Di Bari L, Gandin V, Evangelisti C, Vitulli G, Schiavi E, Marzano C, Ferretti AM, Salvadori P (2015) Glucose-coated superparamagnetic iron oxide nanoparticles prepared by metal vapour synthesis are electively internalized in a pancreatic adenocarcinoma cell line expressing GLUT1 transporter Plos One 10:e0123159. doi: 10.1371/journal.pone.0123159 
Bruvera IJ, Mendoza Zélis P, Pilar Calatayud M, Goya GF, Sánchez FH (2015) Determination of the blocking temperature of magnetic nanoparticles: The good, the bad, and the ugly. J Appl Phys 118:184304. doi:10.1063/1.4935484

Campisi S, Palliggiano S, Gervasini A, Evangelisti C (2019) Finely Iron-Dispersed Particles on beta Zeolite from Solvated Iron Atoms: Promising Catalysts for $\mathrm{NH}_{3}-\mathrm{SCO}$. J Phys Chem C 123:11723-11733. doi: 10.1021/acs.jpcc.9b01474

Coey JMD (2010) Magnetism and Magnetic Materials, Cambridge, Cambridge University Press, p. 266.

Della Pina C, Rossi M, Ferretti AM, Ponti A, Lo Faro M, Falletta E (2012) One-pot synthesis of polyaniline $/ \mathrm{Fe}_{3} \mathrm{O}_{4}$ nanocomposites with magnetic and conductive behaviour. Catalytic effect of $\mathrm{Fe}_{3} \mathrm{O}_{4}$ nanoparticles. Synth Met 162:2250-2258. doi: 10.1016/j.synthmet.2012.10.023; Della Pina C, Falletta E, Ferretti AM, Ponti A, Gentili GG, Verri V, Nesti R (2014) Microwave characterization of magnetically hard and soft ferrite nanoparticles in K-band. J Appl Phys 116:154306. doi:10.1063/1.4898138; Della Pina C, Ferretti AM, Ponti A, Falletta E (2015) A green approach to magnetically-hard electrically-conducting polyaniline/ $\mathrm{CoFe}_{2} \mathrm{O}_{4}$ nanocomposites. Compos Sci Technol 110:138-144. doi: 10.1016/j.compscitech.2015.02.007; Della Pina C, De Gregorio MA, Clerici L, Dellavedova P, Falletta E (2018) Polyaniline (PANI): an innovative support for sampling and removal of VOCs in air matrices. J Hazard Mater 344:1-8. doi: 10.1016/j.jhazmat.2017.10.012

Dong H, Hu W (2012) Conducting Polymers: Applications in Electronics and Photovoltaics in Book: Encyclopedia of Radicals in Chemistry, Biology and Materials, Publisher: John Wiley and Sons.

Evangelisti C, Schiavi E, Aronica LA, Psaro R, Balerna A, Martra G (2015) Solvated Metal Atoms in the Preparation of Supported Gold Catalysts. In: Prati L, Villa A (eds), Gold Catalysis: Preparation, Characterization and Applications, Jenny Stanford Publishing, Beijing, PRC.

Falletta E, Ponti A, Sironi A, Ferretti AM, Della Pina C (2015) Nanoferrites as Catalysts and Fillers for Polyaniline/Nanoparticle Composites Preparation. J Adv Catal Sci Technol 2:8-16. doi: 10.15379/2408-9834.2015.02.02.02

Ferretti AM, Usseglio S, Mondini S, Drago C, La Mattina R, Chini B, Verderio C, Leonzino M, Cagnoli C, Joshi P, Boraschi D, Italiani P, Li Y, Swartzwelter BJ, Sironi L, Gelosa P, Castiglioni L, Guerrini U, Ponti A (2021) Towards bio-compatible magnetic 
nanoparticles: Immune-related effects, in-vitro internalization, and in-vivo biodistribution of zwitterionic ferrite nanoparticles with unexpected renal clearance. $\mathrm{J}$ Coll Interf Sci 582:678-700. doi:10.1016/j.jcis.2020.08.026.

Fiorillo F (2010) Measurements of magnetic materials. Metrologia 47:S114-S142. doi:10.1088/0026-1394/47/2/S11

Hyeon T, Lee SS, Park J, Chung Y, Na H B (2001) Synthesis of highly crystalline and monodisperse maghemite nanocrystallites without a size-selection process. J Am Chem Soc 123:12798-12801. doi:10.1021/ja016812s

Ibanez JG, Rincón ME, Gutierrez-Granados S, Chaha M, Jaramillo-Quintero OA, FrontanaUribe BA (2018) Conducting Polymers in the Fields of Energy, Environmental Remediation, and Chemical-Chiral Sensors. Chem Rev 118(9):4731-4816. doi: 10.1021/acs.chemrev.7b00482

Kaur G, Adhikari R, Cass P, Bown M, Gunatillake P (2015) Electrically conductive polymers and composites for biomedical applications. RSC Adv 5:37553-37567. doi: 10.1039/C5RA01851J

Li J, Qiao J, Lian K (2020) Hydroxide ion conducting polymer electrolytes and their applications in solid supercapacitors: A review. Energy Stor. Mater 24:6-21. doi: 10.1016/j.ensm.2019.08.012

Mišurović J, Mojović M, Marjanović B, Vulić P, Ćirić-Marjanović G (2019) Magnetite nanoparticles-catalysed synthesis of conductive polyaniline. Synth Met 257:116174(111). doi:10.1016/j.synthmet.2019.116174

Mondini S, Ferretti AM, Puglisi A, Ponti, A (2012) PEBBLES and PEBBLEJUGGLER: Software for Accurate, Unbiased, and Fast Measurement and Analysis of Nanoparticle Morphology from Transmission Electron Microscopy (TEM) Micrographs. Nanoscale 4:5356-5372. doi:10.1039/C2NR31276J

Mondini S, Leonzino M, Drago C, Ferretti AM, Usseglio S, Maggioni D, Tornese P, Chini B, Ponti A (2015) Zwitterion-Coated Iron Oxide Nanoparticles: Surface Chemistry and Intracellular Uptake by Hepatocarcinoma (HepG2) Cells. Langmuir 31:7381-7390. doi:10.1021/acs.langmuir.5b01496

Movassagh-Alanagh F, Bordbar-Khiabani A, Ahangari-Asl A (2017) Three-phase PANI@nano-Fe30 $\mathrm{O}_{4} @ \mathrm{CFs}$ heterostructure: Fabrication, characterization and investigation of microwave absorption and EMI shielding of PANI@nano$\mathrm{Fe}_{3} \mathrm{O}_{4} @$ @ Fs/epoxy hybrid composite. Compos Sci Technol 150:65-78. doi: 10.1016/j.compscitech.2017.07.010 
653 Muhammad A, Shah AHA, Bilal S, Rahman G (2019) Basic Blue Dye Adsorption from Water Using Polyaniline/Magnetite $\left(\mathrm{Fe}_{3} \mathrm{O}_{4}\right)$ Composites: Kinetic and Thermodynamic Aspects. Materials,12:1764(1-26). doi: 10.1039/C7SE00139H10.3390/ma12111764

Park J, An K, Hwang Y, Park J-G, Noh H-J, Kim J-Y, Park J-H, Hwang N-M, Hyeon T (2004) Ultra-large-scale syntheses of monodisperse nanocrystals. Nature Mater 3:891-895. doi:10.1038/nmat1251

Qiu G, Wang Q, Nie M (2006) Polyaniline/ $/ \mathrm{Fe}_{3} \mathrm{O}_{4}$ Magnetic Nanocomposite Prepared by Ultrasonic Irradiation. J Appl Polym Sci 102:2107-2111. doi: 10.1002/app.24100

Tzou K, Gregory RV (1992) Kinetic study of the chemical polymerization of aniline in aqueous solutions. Synth Met 47:267-277. doi:10.1016/0379-6779(92)90367-R

Wang X, Liu Y, Han H, Zhao Y, Mad W, Sun H (2017) Polyaniline coated $\mathrm{Fe}_{3} \mathrm{O}_{4}$ hollow nanospheres as anode materials for lithium ion batteries. Sustain. Energy Fuels 1:915922. doi: 10.1039/C7SE00139H

Wang Y, Liu A, Han Y, Li T (2020) Sensors based on conductive polymers and their composites: a review. Polym Int, 69:7-17. doi:10.1002/pi.5907

Woińska M, Szczytko J, Majhofer A, Gosk J, Dziatkowski K, Twardowski A (2013) Magnetic interactions in an ensemble of cubic nanoparticles: A Monte Carlo study. Phys Rev B 88:144421. doi:10.1103/PhysRevB.88.144421

Yang C, Zhang P, Nautiyal A, Li S, Liu N, Yin J, Deng K, Zhang X (2019) Tunable ThreeDimensional Nanostructured Conductive Polymer Hydrogels for Energy-Storage Applications. ACS Appl Mater Interfaces 11(4):4258-4267. doi: 10.1021/acsami.8b19180

Yun H, Liu X, Paik T, Palanisamy D, Kim J, Vogel WD, Viescas AJ, Chen J, Papaefthymiou GC, Kikkawa JM, Allen MG, Murray CB (2014) Size- and Composition-Dependent Radio Frequency Magnetic Permeability of Iron Oxide Nanocrystals. ACS Nano 8:12323-12337. doi:2481/10.1021/nn504711g. 
681 Graphical Abstract

682

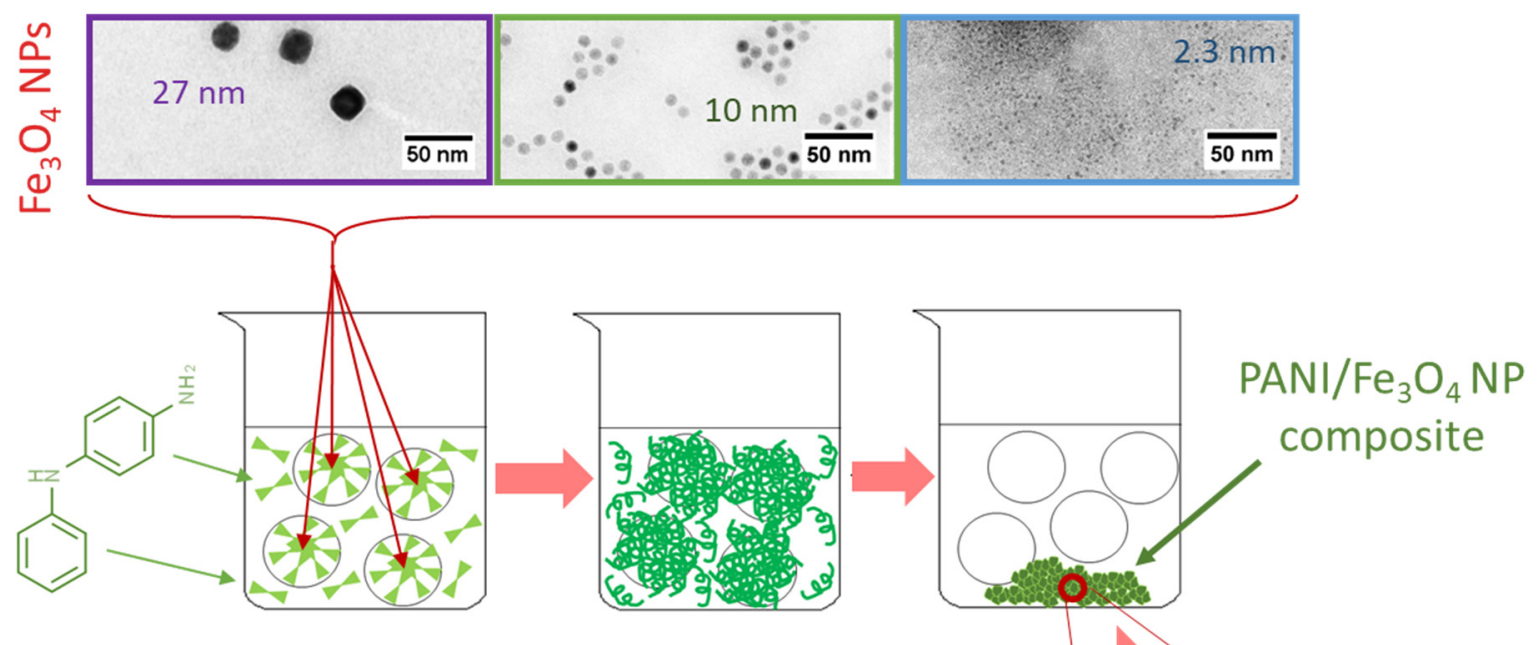

Nanoparticle-catalyzed oxidative polymerization
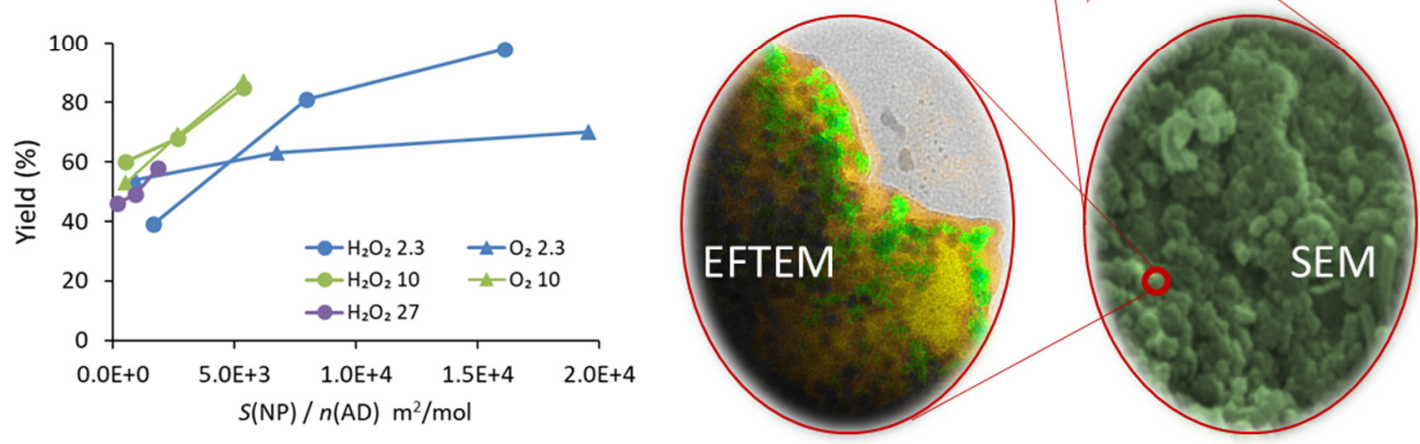